\title{
Anhang 8: Standorte der einzelnen Teilnachlässe heute
}

\section{Houghton Library / Harvard College Library, Cambridge (MA)}

Die Houghton Library bewahrt den größten Teil der wissenschaftlichen Manuskripte, der wissenschaftlichen Korrespondenz sowie einen kleinen, aber wichtigen Teil der Familienkorrespondenz auf. Insgesamt hat die Brentano-Sammlung der Houghton Library einen Umfang von rund 38.000 Seiten, wobei 23.000 Seiten auf die Werkmanuskripte und 15.000 Seiten auf die mehr als 3.000 Briefe $^{1}$ entfallen. Sowohl von den Werkmanuskripten als auch von den Briefen gibt es Verzeichnisse, die die einzelnen Dokumente beschreiben. ${ }^{2}$ Sämtliche Dokumente wurden digitalisiert und sind auf der Website des FBAG online zugänglich (gams.uni-graz.at/context:bag).

\section{Blonay}

Im Archiv der Familie Brentano befindet sich die umfangreiche Familienkorrespondenz Brentanos, darunter der äußerst umfangreiche Briefwechsel mit seiner Mutter (ca. 900 Briefe). Darüber hinaus enthält das Archiv eine kleine Anzahl wissenschaftlicher Notizen, die eigenhändige Korrekturfahne der $\mathrm{PeS}$, aber auch fast das gesamte literarische Werk von Brentano (Gedichte, Theaterstücke, Übersetzungen). Der Gesamtumfang des Bestandes beträgt fast 20.000 Seiten, wobei rund 16.500 Seiten auf 4.400 Briefe entfallen. Von den Beständen in Blonay existiert ein provisorisches Verzeichnis, das für die Korrespondenz allerdings nur die Korrespondenzpartner, die ungefähre Laufzeit und die Anzahl der Briefe angibt. Das Familienarchiv ist nicht öffentlich zugänglich.

\section{Franz Brentano-Archiv Graz}

Der wichtigste Teil der Brentano-Sammlung in Graz war zunächst Brentanos rund 800 Bände umfassende wissenschaftliche Handbibliothek mit zahlreichen eigenhändigen Annotationen Brentanos und vielen Einlagen mit wissenschaftlichen Notizen. Außerdem verfügte das Archiv über einige wenige Originalmanuskripte (darunter die Habilitationsthesen) sowie Originalbriefe aus der Korrespondenz Brentanos mit Anton Marty und Oskar Kraus.

2012 konnte die Brentano-Sammlung durch eine Schenkung der Oxford University vergrößert werden. Dieses Konvolut enthält neben 34 zum Teil unbekannten Originalmanuskripten Brentanos auch zahlreiche Briefe von Carl Stumpf und Oskar Kraus an Brentano, sowie sämtliche Briefe John Stuart Mills an Brentano.

1 Als „Briefe“ werden hier auch Postkarten oder andere Korrespondenzstücke gezählt.

2 Für die Werkmanuskripte gilt das allerdings nicht in allen Fällen (vgl. oben 293). 
Im Jahr 2016 schließlich konnte das FBAG auch den Nachlass von Alfred Kastil aus Schönbühel übernehmen, der einen relativ umfangreichen Kryptonachlass Brentanos enthält. Dieser besteht neben zahlreichen kurzen wissenschaftlichen Notizen und diversen Briefen an Brentano auch aus zwei umfangreicheren Werkmanuskripten sowie den handschriftlichen Druckvorlagen zu Brentanos Dissertations- und Habilitationsschrift und zu drei Publikationen aus dem Jahr 1911. Insgesamt enthält der Kryptonachlass fast 200 Manuskripte wissenschaftlicher und nichtwissenschaftlicher (Rätsel, Schachschriften) Natur und rund 480 Briefe (360 an Brentano, 30 Briefe von Brentano). Der Kryptonachlass aus Schönbühel ist in Einzelaufnahme erschlossen und fast vollständig digitalisiert, ${ }^{3}$ von den beiden anderen Sammlungen am FBAG existieren zurzeit lediglich provisorische Verzeichnisse. Die Digitalisate sollen in naher Zukunft online zugänglich gemacht werden.

\section{Freies Deutsches Hochstift / Goethe-Haus Frankfurt}

Das Freie Deutsche Hochstift sammelt bevorzugt die Nachlässe der deutschen Romantiker, weshalb auch der Nachlass von Brentanos Onkel Clemens Brentano hier aufbewahrt und kritisch herausgegeben wird. Daneben gibt es weiteres umfangreiches Material zu den Frankfurter und Aschaffenburger Brentanos. Der Bestand „Franz Brentano“ enthält neben einigen Gedichten insgesamt 79 Briefe, 7 Briefe von Brentano und 72 Briefe an ihn. Bei den Korrespondenzpartnern handelt es sich hauptsächlich um Familienmitglieder bzw. um der Familie nahestehende Personen.

\section{Stadt- und Stiftsarchiv Aschaffenburg}

Die Aschaffenburger Brentano-Sammlung enthält zahlreiche Korrespondenzen und Lebensdokumente der Frankfurter und Aschaffenburger Brentanos. Neben einigen Lebensdokumenten wird ein kleiner Teil der Korrespondenz Brentanos mit seinem Bruder Lujo (8 Briefe von Franz, 7 Briefe von Lujo; 1880 bis 1913) im Stadt- und Stiftsarchiv aufbewahrt. Der größte Teil der Aschaffenburger Sammlung ist jedoch Emilie Brentano-Genger, Lujo und seiner Tochter Sissi Brentano zuzuordnen. Der Bestand wurde 2014 neu katalogisiert, wobei allerdings kaum Einzelaufnahmen gemacht wurden.

\section{Verstreutes}

Seit den Aktivitäten des Prager Brentano-Archivs sind keine nennenswerten systematischen Anstrengungen mehr unternommen worden, Briefe Brentanos in den Nachlässen seiner Korrespondenzpartner auszuforschen. Dass solche Dokumente

3 Das Verzeichnis ist auch online auf der Website des FBAG zugänglich. 
tatsächlich noch existieren, ergaben stichprobenartige Recherchen in den Beständen der Österreichischen Nationalbibliothek und der Wienbibliothek (der ehemaligen Wiener Stadt- und Landesbibliothek), bei denen z. B. Briefe Brentanos an die Dichterin Marie von Ebner-Eschenbach und die Schriftstellerin Hermine Cloeter entdeckt wurden. Die Briefe Brentanos an Masaryk befinden sich im Masaryk-Archiv in Prag, Brentanos Briefe an Alexius Meinong in der Sondersammlung der Universitätsbibliothek Graz, und Brentanos Briefe an Kazimierz Twardowski im Twardowski-Archiv in Warschau.

8. Weitere Sammlungen mit Brentano-Bezug

Universitätsarchiv Innsbruck

Franz Brentano Forschung Würzburg

Tschechische Akademie der Wissenschaften Prag 